\title{
Is there a link between sensory alterations and modification in food preference following bariatric surgery?
}

\author{
E. Guyot ${ }^{1,2}$, A. Dougkas ${ }^{2}$, J.A. Nazare ${ }^{1}$, S. Iceta ${ }^{3}$ and E. Disse ${ }^{1,4}$ \\ ${ }^{1}$ Center for Research in Human Nutrition Rhône-Alpes (CRNH Rhône-Alpes), Univ Lyon, CarMeN Laboratory, \\ INSERM, INRA, INSA Lyon, Université Claude Bernard Lyon 1, 69130, Pierre-Bénite, France, \\ ${ }^{2}$ Institut Paul Bocuse Research Center, 69130, Ecully, France, \\ ${ }^{3}$ Centre de Recherche de l'Institut Universitaire de Cardiologie et de Pneumologie de Québec-Université Laval, 2725, \\ Chemin Sainte-Foy, Quebec, QC G1V 4G5, Canada and \\ ${ }^{4}$ Hospices Civils de Lyon, Integrated Center for Obesity, Department of Endocrinology and Diabetes, Lyon-Sud \\ Hospital, 69310, Pierre-Bénite, France
}

While changes in food preferences and sensory alterations have been reported after bariatric surgery independently, limited studies explored the relationship between food preferences and sensory alterations at the same cohort following bariatric surgery. Thus, we aimed to assess the link between food preferences, and taste and smell alterations among patients with different types of bariatric surgery.

In this cross-sectional study, we used an online questionnaire to assess current preferences and perceived changes in preferences of 24 foods compared to before the bariatric surgery using liking scales. Patients also reported perceived qualitative and quantitative changes in taste and smell. Statistical analysis involved Mann-Whitney $U$ test.

Two hundred twenty patients answered the questionnaire (women: 92\%; age: $42 \pm 11 \mathrm{y}$; preoperative body mass index (BMI): $44 \pm 6$ kg.m-2; current BMI: $30 \pm 7$ kg.m-2; excess weight loss: $76 \pm 31 \%$; sleeve: $56 \%$; gastric bypass: $32 \%$; other surgeries: $12 \%$; follow up: 1.3(0-19)y). The liking scores were higher for vegetables, cheese and fruits and lower for fried foods, sugary drinks, and alcohol. Overall, patients reported a decreased appreciation for high-fat and high-carb foods as well as red meat, and an increased appreciation for fruits, vegetables and fish compared to before the bariatric surgery. Among the $64 \%$ of patients with taste alteration, the majority had hypergeusia (53\%). Smell alteration was reported by $38 \%$ of the patients, who had mostly hyperosmia ( $77 \%$ ). Only $33 \%$ of the patients had no reported taste or smell alteration. Compared to before the surgery, patients with taste alteration reported a decreased preference for red meat, dessert, fried foods and fat as well as an increased preference for green vegetables (all $\mathrm{p}<0.05)$. Those with smell alteration reported a decreased preference for red meat, eggs, sweet dairy products and fat $($ all $\mathrm{p}<0.05)$.

This study suggests that food preferences differ between patients with compared to those patients without taste and smell alterations. For those who experienced sensory alterations, there was a decreased preference for unhealthy food items. Further research is needed to confirm these results and to examine other factors that could be involved in these modifications, including food intolerance and food reward. 